\title{
PAPER
}

\section{A novel quality of life instrument for deep brain stimulation in movement disorders}

\author{
A Kuehler, G Henrich, U Schroeder, B Conrad, P Herschbach, A Ceballos-Baumann
}

J Neurol Neurosurg Psychiatry 2003;74:1023-1030

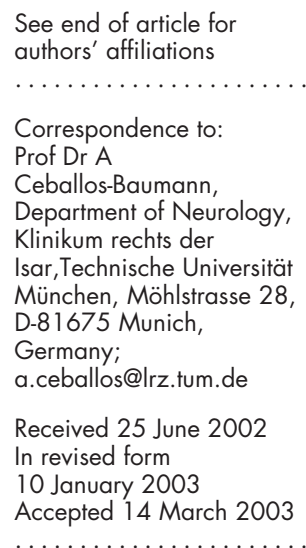

\begin{abstract}
Objective: To develop a short instrument to examine quality of life (QoL) which specifically addresses patients with movement disorders treated by deep brain stimulation (DBS).

Design: The instrument was developed within an existing concept of a modular questionnaire (questions on life satisfaction: "general life satisfaction" QLS"-A, and "satisfaction with health" QLS $-G$ ), in which each item is weighted according to its relative importance to the individual.

Methods: Items were generated by interviews with 20 DBS patients, followed by item reduction and scale generation, factor analysis to determine relevant and final questionnaire items, estimation of reliability, and validation based on the medical outcome study 36 item short form health survey (SF-36) and the EuroQol (EQ-5D) (data from 152 patients with Parkinson's disease, essential tremor, or idiopathic torsion dystonia, including 75 patients with DBS).

Results: Initial questionnaires were reduced to 12 items for a "movement disorder module" (QLSM-MD), and five items for a "deep brain stimulation module" (QLS"-DBS). Psychometric analysis revealed Cronbach's $\alpha$ values of of 0.87 and 0.73 , and satisfactory correlation coefficients for convergent validity with SF-36 and EQ-5D.

Conclusions: QLS $-M D$ and QLS ${ }^{M}-D B S$ can evaluate quality of life aspects of DBS in movement disorders. Psychometric evaluation showed the questionnaires to be reliable, valid, and well accepted by the patients.
\end{abstract}

M ovement disorders have a strong impact on patients' quality of life (QOL). Disability, immobility, social handicap, dependency, and stigmatisation have been found using generic QoL instruments to represent the major factors contributing to poor quality of life in essential tremor, ${ }^{1}$ idiopathic torsion dystonia, ${ }^{23}$ and Parkinson's disease. $^{4}$

Deep brain stimulation (DBS) is increasingly used as a therapeutic option in movement disorders. ${ }^{5-9}$ Quality of life becomes increasingly important as a crucial variable for clinical trials, ${ }^{10}$ particularly for expensive treatment options in chronic disease. DBS results in increased costs (total medical cost, €28 920) compared with drug treatment alone and is restricted by health care providers. ${ }^{11}$ The main DBS studies have focused on clinical features and activities of daily living in movement disorders. ${ }^{5-9}$ DBS has been shown to improve quality of life in Parkinson's disease and essential tremor, using generic QoL instruments in small studies. In Parkinson's disease, improvements were noted for social interaction, communication, alertness, and emotional behaviour. ${ }^{12}$ Patients with essential tremor rated themselves as better able to communicate and felt less stigmatised..$^{13}$ However, the subjective significance of the disabilities and the special situation of patients with an implanted programmable electrical brain device cannot be taken into account with existing instruments. Apart from its beneficial effects on akinesia, tremor, and dystonia, DBS may have adverse effects which are either associated with the surgical procedure itself (for example, intracranial haemorrhage, scars, disconnection of the system), or are related to stimulation (dysarthria, dysequilibrium, dysaethesiae) or to the device (programming failures, inadvertent switching off by electrical razors, metal detectors, and so on, and problems related to switching DBS on and off, or changing the amplitude). Adverse events related to the procedure or to the stimulation may result in transient deficits or long term sequelae which can have adverse effects on quality of life. Patients may also have misleading fantasies and fears of wearing a controllable brain implant which could interfere with treatment satisfaction and compliance. Often, frequent appointments are necessary to adjust stimulator settings and to change drug treatment accordingly.

A QoL instrument that includes specific DBS related aspects would be helpful to justify DBS over conservative treatment, if it could be shown to result in a significantly better quality of life outcome; and to identify the type of patient who would have the best chance of subjective satisfaction with DBS treatment. Our aim was to develop a short instrument to examine quality of life which takes account of the special circumstances of patients with movement disorders treated with DBS. Our study included patients with Parkinson's disease, essential tremor, and generalised dystonia-all movement disorders that are commonly treated with DBS. The instrument was based on an existing and well established multidimensional questionnaire concept, the "questions on life satisfaction $^{\text {modules" }}\left(\mathrm{QLS}^{\mathrm{M}}\right){ }^{14}$ Two new modules, one for "movement disorder" (QLS ${ }^{\mathrm{M}}$-MD) and one for "deep brain stimulation" $\left(\right.$ QLS $^{\mathrm{M}}$-DBS) are presented, which supplement the two basic modules of "general life satisfaction" (QLS ${ }^{\mathrm{M}}$-A) and "satisfaction with health" (QLS $\left.{ }^{M}-G\right)$. A key feature of this modular questionnaire concept-distinguishing it from other disease specific, self rating questionnaires-is that each item is weighted according to its relative importance to the individual. ${ }^{15}$ The questionnaires are reproduced in figs 1 and 2 .

Abbreviations: DBS, deep brain stimulation; EQ-5D, EuroQol survey; PCA, principal component analysis; QLS ${ }^{M}$, questions on life satisfaction ${ }^{\text {modules; }}$ QLSM-A, questions on life satisfaction, general satisfaction; $Q L S^{M}-G$, questions on life satisfaction, satisfaction with health; QLS ${ }^{M}-D B S$, questions on life satisfaction, deep brain stimulation; QLSM-MD, questions on life satisfaction, movement disorder; QoL, quality of life; SF-36, medical outcome study 36 item short form health survey 

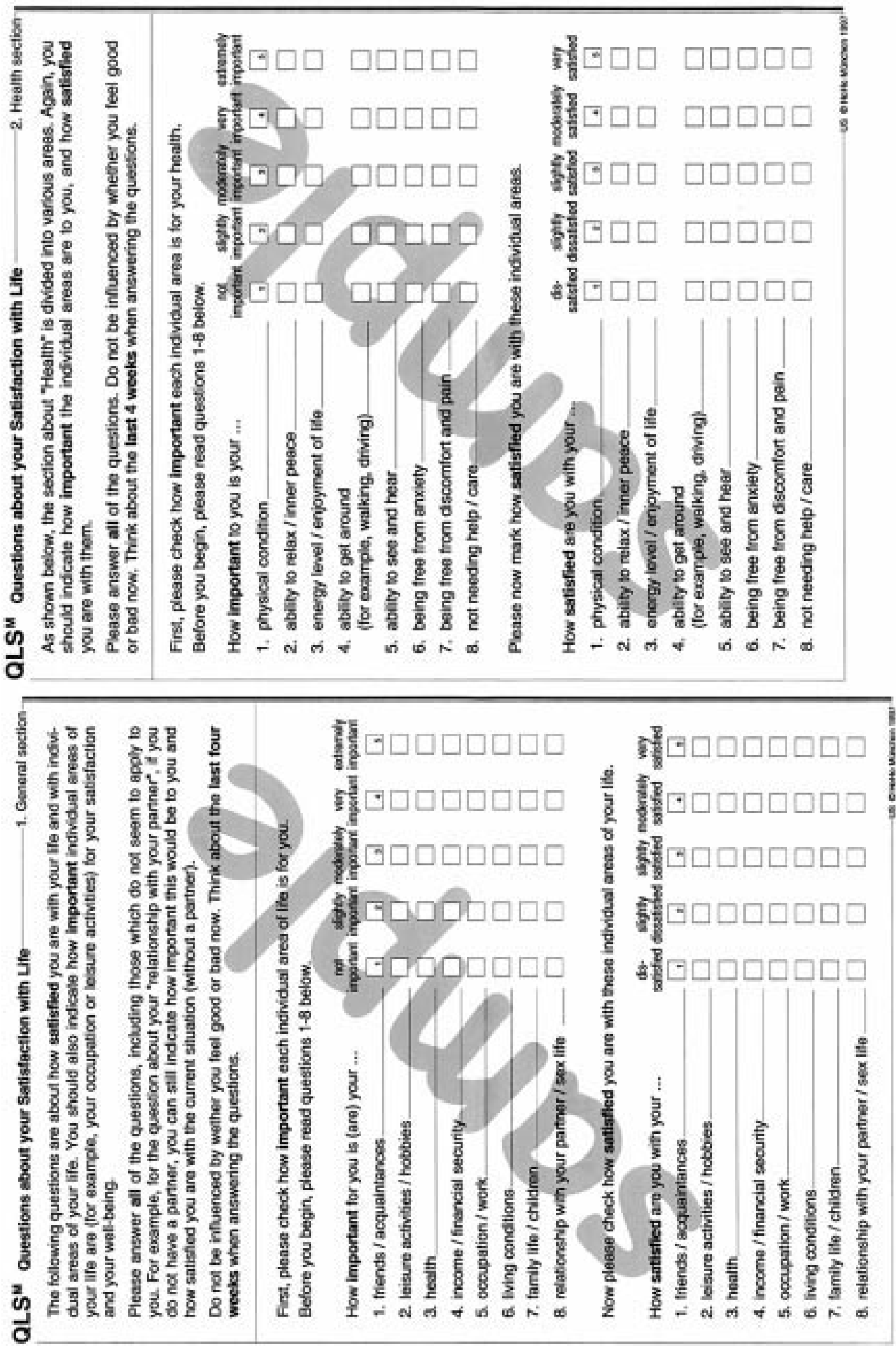

Figure 1 Original quality of life questionnaire $\left(Q L S^{M}\right)$

\section{METHODS}

\section{Item generation}

In this step, exploratory interviews were carried out in 20 patients who were having DBS for movement disorders caused by Parkinson's disease $(n=12)$, essential tremor $(n=5)$, and idiopathic torsion dystonia $(\mathrm{n}=3)$. Patients were recruited through our outpatient clinic for movement disorders. Selected individuals with the greatest possible variety of age, socioeconomic status, and the presenting stage of the movement disorder were asked to describe areas of their lives 

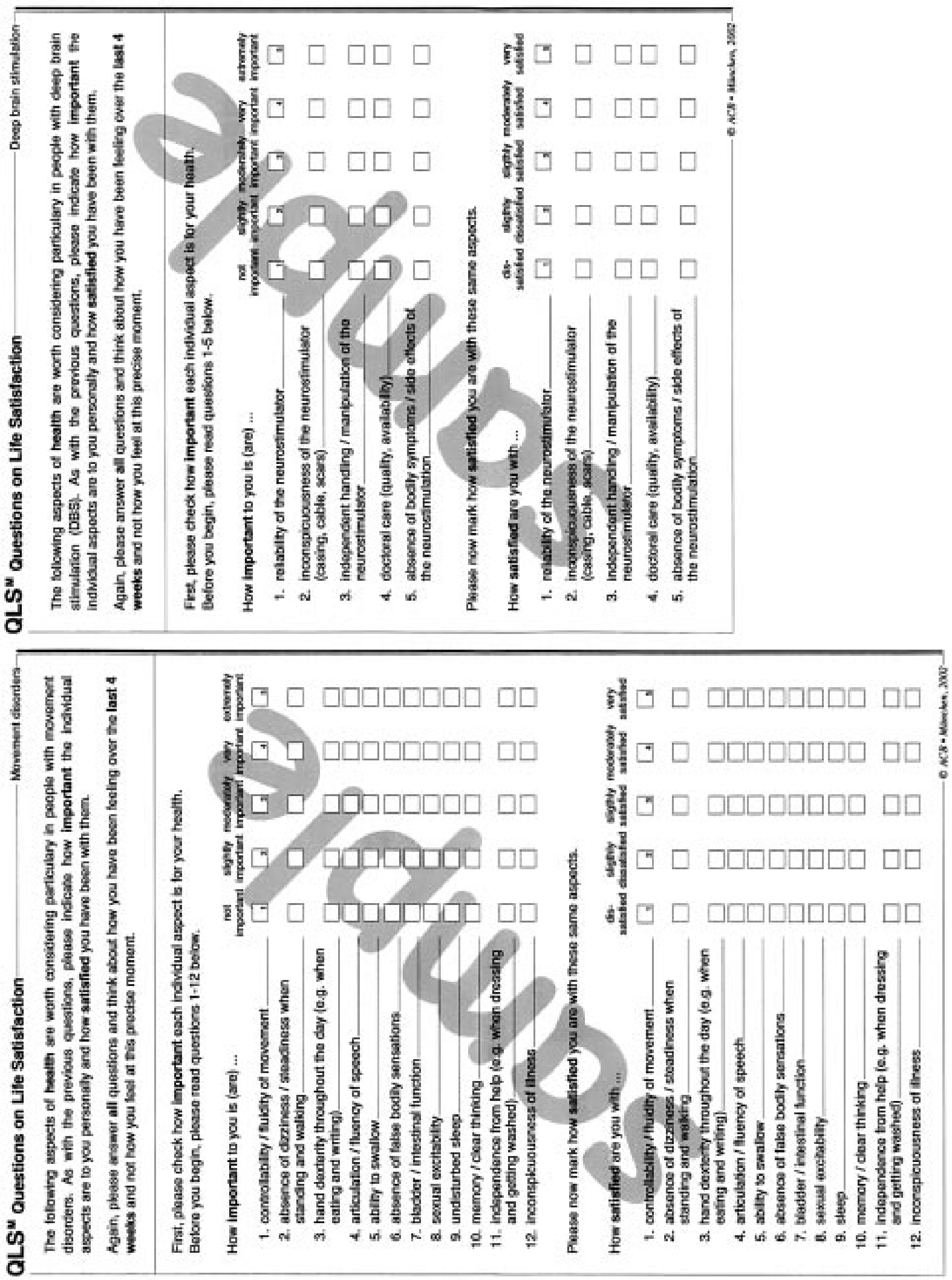

Figure 2 The new modules

that had been influenced first by their disease, and second by deep brain stimulation. All patients had a running deep brain stimulator, and showed different degrees of responsiveness and satisfaction with treatment. An interview lasted about 90 to 120 minutes. On the basis of a literature research and clini- cal experience, life areas addressed were "general life", "physical fitness and abilities", "concentration and memory", "friends, contacts and leisure activities", "job, housekeeping and everyday life", "family, relationships, and sexuality", "feelings and mood", and "treatment contentment and 
stimulator handling." In the semistructured interview we presented open questions, for example, "How does your illness/your neurostimulator affect your physical fitness and abilities at the moment?" These were followed by formulated proposals to be answered by yes or no-for example, "Are you able to stand physical stress?", "Do you feel nerveless and weak?", and so on. After intensive consideration of a life area with the interviewer, patients were invited to consider the completeness of their answers, as follow: "Concerning your physical fitness and abilities, are there any other effects of your illness/of neurostimulation which we have not discussed so far?" A significant number of categories in which quality of life was restricted were reported. We had to deal with many possible questionnaire items. These data were inspected for overlapping, ambiguity, and repetition. In the formulation of items, all signs, symptoms, and disabilities were neutrally rephrased to ensure that they could be answered in either a positive or a negative direction. An additional linguistic review resulted in a preliminary 29 item questionnaire for the "movement disorder module" and a 24 item questionnaire for the "neurostimulation module." Global format, instructions, and answer categories of the preliminary questionnaires were similar to those in the established QLS $^{\mathrm{M}}$ (see figs 1 and 2). Basic acceptability and comprehension of the two preliminary modules were tested by two patients from a local self help group for Parkinson's disease and DBS, two psychologists, and medical staff from the outpatient clinic.

\section{Item reduction and scale generation}

The preliminary questionnaires which were generated in the step described above were posted to a sample of individuals with Parkinson's disease, essential tremor, or idiopathic torsion dystonia. Patients without deep brain stimulation (non-DBS) received the 29 item questionnaire for movement disorders. The latter questionnaire plus the 24 item questionnaire for neurostimulation were send to patients with deep brain stimulators for movement disorders. In this phase of development the suitability of the instrument was tested. This stage also allowed us to develop shorter modules with a reduced number of items.

Patients were recruited through the German Parkinson's Disease Society, the German Dystonia Society, a local essential tremor self help group, and our outpatient clinic. Each participant gave informed consent. In all, 216 questionnaires were posted out ( 113 to patients with DBS); 152 individual sufficiently completed and returned the questionnaires (102 with Parkinson's disease, 26 with essential tremor, and 24 with idiopathic torsion dystonia, including 75 with DBS). The response rate was $70.4 \%$. The mean (SD) age of the respondents was 58.4 (16.3) years for non-DBS patients, and 62.8 (10.5) years for DBS patients, with a male to female ratio of $1: 1.3$ and $2.2: 1$, respectively. The mean age at onset of disease was $46.4(21.4)$ years in respondents without DBS $v 44.0$ (13.6) years in the DBS group. The mean period since implantation of the brain stimulator device was 37.1 (29.1) months $(\mathrm{n}=74)$. The data revealed by this postal survey were factor analysed, applying principal component analysis (PCA) with subsequent Oblimin rotation to establish the essential and underlying dimensions of the two preliminary questionnaires for movement disorders and neurostimulation. Psychometric evaluation was done by reliability testing and validation with established instruments (medical outcome study 36 item short form health survey (SF-36) ${ }^{16}$ and the EuroQol (EQ-5D) ${ }^{17}$ ).

\section{RESULTS}

\section{Factor analysis}

The aim of this step of the questionnaire development was to establish a final set of items that contains minimum redundancy, for economic reasons, and which also complies with the principal psychometric quality criteria. In such cases, factor analysis is a common approach for identifying highly intercorrelated items. ${ }^{18}$ As factor analysis requires complete data records, the missing data were replaced by an estimated value (expectation maximisation algorithm) with the help of the SPSS missing value analysis. ${ }^{19}$ The item means of the movement disorder module ( -1.2 to 8.7 ; SD 5.2 to 9.3) were relatively close to the median of the possible range of values $(-12$ to 20$)$. The corresponding values for the DBS module were: mean between 0.8 and 11.3, SD between 4.5 and 9.2. The distribution of only eight of 46 items deviated significantly from the normal distribution (Kolmogorof Smirnov test; $p<0.001)$. We have therefore complied with the prerequisites of an explorative factor analysis sufficiently. In order to minimise redundancy in the list of 29 items for movement disorders, similar items were grouped by exploratory PCA with subsequent oblique rotation. As a result of the PCAs, nine factors were selected. This selection was based on the plot of the Eigen values against the number of factors. Assignment of items to the factors was done according to the the highest factor loadings. The first factor, which initially covered nine items, was clinically heterogeneous. A further PCA of this factor revealed three new, relevant, and clinically interpretable factors: "controllability/fluidity of movement", "inconspicuousness of illness", and "absence of false bodily sensations." In another factor we deviated from the initial PCA result for clinical reasons. The item "ability to swallow" had a factor loading of 0.74 within a factor containing items for eating, drinking, writing, and using the telephone. We decided to group the latter items together as "hand dexterity throughout the day (for example, when eating and writing)." "Ability to swallow" was used separately as a single item to acknowledge its clinical significance in movement disorders.

In the final analysis, PCA of the movement disorder items led to 12 factors which form the items of the final questionnaire QLS ${ }^{M}-\mathrm{MD}$. Table 1 shows the final and primary items together with their factor loadings. They can be interpreted as correlations of items with their factor totals:

(1) controllability/fluidity of movement (originally six items);

(2) absence of dizziness/steadiness when standing and walking (two items);

(3) hand dexterity throughout the day (four items);

(4) articulation/fluency of speech (two items);

(5) ability to swallow (one item);

(6) absence of false bodily sensations (one item);

(7) bladder/intestinal function (two items);

(8) sexual excitability (two items);

(9) undisturbed sleep (one item);

(10) memory/clear thinking (two items);

(11) independence from help (four items);

(12) inconspicuousness of illness (two items).

To prevent overlapping of the two modules QLS ${ }^{\mathrm{M}}$-MD and QLS $^{\mathrm{M}}$-DBS and to ensure that the instruments were short, in QLS $^{\mathrm{M}}$-DBS we omitted all items which could be both a side effect of neurostimulation and also a symptom of a movement disorder. Excluded were "relaxed musculature," "steadiness when standing and walking," "fear to fall," and "fluency of speech." These items are already sufficiently covered by the movement disorder module. "Weight" and "visual acuity" were also excluded, because these items were non-specific features for neurostimulation. (Some cases of weight gain during DBS have been reported, but in most cases weight gain was a desired effect in previously emaciated patients.) These exclusions were done before calculating the PCA. The content of these primary items should be covered by the new item "absence of bodily symptoms/side effects of the neurostimulation." The PCA identified five relevant factors from the 
Table 1 Factors and item to total correlation of QLS"-MD, "movement disorder" questionnaire

\begin{tabular}{|c|c|c|}
\hline \multicolumn{3}{|c|}{ QLSM-MD “movement disorder" } \\
\hline Factor & Item & Factor loading \\
\hline \multicolumn{3}{|c|}{ (1) Controllability/fluidity of movement } \\
\hline & Promptness of movement & 0.89 \\
\hline & Keep movements under control & 0.88 \\
\hline & Fluency of movements & 0.85 \\
\hline & Extent of movement disorder under psychological strain & 0.79 \\
\hline & Relaxed musculature & 0.75 \\
\hline & Predictability of mobility & 0.73 \\
\hline \multicolumn{3}{|c|}{ (2) Absence of dizziness/steadiness when standing and walking } \\
\hline & Feel free from giddiness & 0.81 \\
\hline & Safety of gait & 0.65 \\
\hline \multicolumn{3}{|c|}{ (3) Hand dexterity throughout the day (eg, when eating and writing) } \\
\hline & Ability to eat & 0.87 \\
\hline & Ability to drink & 0.80 \\
\hline & Ability to write & 0.78 \\
\hline & Ability to phone & 0.73 \\
\hline \multicolumn{3}{|c|}{ (4) Articulation/fluency of speech } \\
\hline & Express oneself in a comprehensible way & 0.94 \\
\hline & Express oneself in a confident way & 0.93 \\
\hline (5) Abil & & * \\
\hline (6) Abse & bodily sensations & 0.81 \\
\hline \multicolumn{3}{|c|}{ (7) Bladder/intestinal function } \\
\hline & Regular bowel function & 0.77 \\
\hline & Controlled bladder function & 0.66 \\
\hline \multicolumn{3}{|c|}{ (8) Sexual excitability } \\
\hline & Sexual excitability & 0.89 \\
\hline & Self esteem & 0.54 \\
\hline (9) Und & & 0.86 \\
\hline \multicolumn{3}{|c|}{ (10) Memory/clear thinking } \\
\hline & Extent and dimension of hallucinations & 0.73 \\
\hline & Concentration and memory & 0.60 \\
\hline \multicolumn{3}{|c|}{ (1 1) Independence from help (eg, when dressing and getting washed) } \\
\hline & Ability to dress & 0.94 \\
\hline & Ability to wash & 0.93 \\
\hline & Ability to walk alone without fear to fall & 0.66 \\
\hline & Independence of medicaments & 0.54 \\
\hline \multicolumn{3}{|c|}{ (12) Inconspiciuousness of illness } \\
\hline & Stand out in public & 0.87 \\
\hline & Feeling sure in public & 0.75 \\
\hline
\end{tabular}

remaining 17 items. The factors and items of the final questionnaire were:

(1) reliability of neurostimulator (originally four items);

(2) inconspicuousness of neurostimulator (three items);

(3) independent handling/manipulation of the neurostimulator (three items);

(4) physicians care (three items);

(5) absence of bodily symptoms/side effects of the neurostimulation (four items).

The factors, their underlying items, and the factor loadings are presented in table 2. In order to cover all aspects of movement disorders and DBS related aspects, we recommend that both modules should be used in a package. QLS ${ }^{\mathrm{M}}$-DBS is a supplementary element of QLS ${ }^{M}$-MD.

The results were two new modules fitting into the module based concept of questions on life satisfaction (QLS modules): a 12 item questionnaire for "movement disorders" (QLS $\left.{ }^{\mathrm{M}}-\mathrm{MD}\right)$ and a five item module covering deep brain stimulation specific features $\left(\mathrm{QLS}{ }^{\mathrm{M}}\right.$-DBS). As in the existing modules for "general life satisfaction" (QLS $\left.{ }^{\mathrm{M}}-\mathrm{A}\right)$ and "satisfaction with health" $\left(\right.$ QLS $\left.^{\mathrm{M}}-\mathrm{G}\right)$, subjective weighting of the items was applied-that is, the respondent rated each item twice, once for the subjective importance of an aspect and once for the degree of satisfaction in that area; the combination of these results in a "weighted satisfaction score." Overall the QLS ${ }^{\mathrm{M}}$ is a self rated questionnaire. Each of the modules consists of a single sheet of paper containing both the instructions and five to 12 items requiring a response. Items should be answered in retrospect over the last four weeks. Both the instructions and the scales used for rating subjective importance (ranging from "not important" to "extremely important") and satisfaction (ranging from "dissatisfied" to "very satisfied") are concise in all four modules, making the whole questionnaire easier to complete.

The scale used for rating satisfaction is asymmetrical, with two negative and three positive responses to choose from. A category "neither satisfied nor dissatisfied" was omitted 


\begin{tabular}{|c|c|c|}
\hline \multicolumn{3}{|c|}{ QLSM-DBS “deep brain stimulation" } \\
\hline Factor & Item & Factor loading \\
\hline \multicolumn{3}{|c|}{ (1) Reliability of the neurostimulator } \\
\hline & Reliability of stimulator & 0.85 \\
\hline & Safety of stimulator in the case of falls & 0.79 \\
\hline & Life span of battery & 0.77 \\
\hline & Safety near magnetic fields & 0.70 \\
\hline \multicolumn{3}{|c|}{ (2) Inconspicuousness of the neurostimulator (casing, cable, scars) } \\
\hline & Inconspicuousness of stimulator casing & 0.97 \\
\hline & Inconspicuousness of stimulator cable & 0.97 \\
\hline & Inconspicuousness of stimulator scars & 0.96 \\
\hline \multicolumn{3}{|c|}{ (3) Independent handling/manipulation of the neurostimulator } \\
\hline & Change stimulator settings (amplitude) oneself & 0.93 \\
\hline & Switch stimulator on and off oneself & 0.90 \\
\hline & Feel safe in handling the stimulator & 0.72 \\
\hline \multicolumn{3}{|c|}{ (4) Medical care (quality, availability) } \\
\hline & Possibility of getting into contact with caring medical staff & 0.91 \\
\hline & Distance to treatment centre & 0.89 \\
\hline & Quality of medical treatment & 0.63 \\
\hline \multicolumn{3}{|c|}{ (5) Absence of bodily symptoms/side effects of the neurostimulation } \\
\hline & Ability to control salivation & 0.82 \\
\hline & Sensation of heat, sweating & 0.80 \\
\hline & Paralysis of tongue & 0.71 \\
\hline & Formication & 0.59 \\
\hline
\end{tabular}

intentionally to force a positive or negative decision. In the evaluation of responses, the ratings for importance and satisfaction are combined to yield information about "weighted satisfaction" (WS), which varies between -12 and +20 . The weighting formula is:

$$
\text { WS }=[\text { importance rating }-1] \times[(2 \times \text { satisfaction rating })-5]
$$

provided both ratings are made on scales ranging from 1 to 5 . The measure of global life satisfaction (LS) (with reference to the content of a given module) is the sum of the WS values. The importance scale of the questionnaire is unipolar (that is, ranging from " $1=$ not important," to " $5=$ extremely important") while the satisfaction scale is bipolar (ranging from " $l$
= dissatisfied," to "5 = very satisfied," with a zero point between the second and third category). Therefore, we subtract 1 from the importance ratings in order that "unimportant" answers are not considered in the total score of the QLS (multiplication with zero). The purpose of the formula "[ $2 \times$ satisfaction -5$]$ " is to transform the zero point of the satisfaction scale to 2.5 (between the second and third category). ${ }^{15}$ For further details see figs 1 and 2 .

\section{Reliability}

Internal consistency as a measure of reliability of the new modules was high: Cronbach's $\alpha$ for the module "movement disorders" was 0.87 . A value of 0.73 was reached for the module

Table 3 Score distribution and item characteristics of the new modules

\begin{tabular}{|c|c|c|c|c|c|c|}
\hline & $\begin{array}{l}\text { Missing } \\
\text { data (\%) }\end{array}$ & $\begin{array}{l}\text { Weighted } \\
\text { satisfact } \\
\text { (mean) }\end{array}$ & $\begin{array}{l}\text { Weighted } \\
\text { satisfact (SD) }\end{array}$ & $\begin{array}{l}\text { Floor effects } \\
(\%)\end{array}$ & $\begin{array}{l}\text { Ceiling } \\
\text { effects (\%) }\end{array}$ & $\begin{array}{l}\text { Internal } \\
\text { consistency }\end{array}$ \\
\hline \multicolumn{7}{|l|}{ QLSM-MD "movement disorder" } \\
\hline (1) Controllability/fluidity of movement & 3.2 & 0.2 & 5.4 & 1.3 & 0.0 & 0.74 \\
\hline (2) Absence of dizziness/steadiness & 3.9 & 1.4 & 7.0 & 4.1 & 1.4 & 0.60 \\
\hline (3) Hand dexterity throughout the day & 3.2 & 5.2 & 6.2 & 0.0 & 0.7 & 0.51 \\
\hline (4) Articulation/fluency of speech & 4.6 & 2.8 & 8.1 & 5.5 & 2.8 & 0.53 \\
\hline (5) Ability to swallow & 5.3 & 7.1 & 7.4 & 0.7 & 7.6 & 0.62 \\
\hline (6) Absence of false bodily sensations & 7.9 & 0.4 & 6.6 & 6.4 & 0.7 & 0.59 \\
\hline (7) Bladder/intestinal function & 3.9 & 3.3 & 6.6 & 1.4 & 1.4 & 0.59 \\
\hline (8) Sexual excitability & 14.4 & 2.2 & 5.2 & 0.0 & 1.5 & 0.38 \\
\hline (9) Undisturbed sleep & 6.5 & 4.4 & 7.6 & 2.1 & 5.6 & 0.36 \\
\hline (10) Memory/clear thinking & 3.9 & 5.4 & 5.9 & 0.7 & 0.7 & 0.61 \\
\hline (11) Independence from help & 3.9 & 3.6 & 6.4 & 1.4 & 0.7 & 0.70 \\
\hline (12) Inconspicuousness of illness & 5.3 & 1.2 & 5.2 & 1.4 & 0.0 & 0.66 \\
\hline Summary index & 3.9 & 37.1 & 51.5 & 0.0 & 0.0 & 0.87 \\
\hline \multicolumn{7}{|l|}{ QLSM-DBS "deep brain stimulation" } \\
\hline (1) Reliability of the neurostimulator & 10.7 & 8.6 & 6.0 & 0.0 & 6.0 & 0.61 \\
\hline (2) Inconspicuousness of the stimulator & 10.7 & 3.0 & 4.5 & 0.0 & 3.0 & 0.34 \\
\hline (3) Independent handling of the stimulator & 12.0 & 3.8 & 6.4 & 0.0 & 1.5 & 0.57 \\
\hline (4) Doctor care (quality, availability) & 12.0 & 3.7 & 7.4 & 3.0 & 17.3 & 0.54 \\
\hline (5) Absence of bodily symptoms & 9.3 & 2.4 & 4.9 & 0.0 & 0.0 & 0.46 \\
\hline Summary index & 10.7 & 21.5 & 20.5 & 0.0 & 0.0 & 0.73 \\
\hline
\end{tabular}


Table 4 Correlation between the new QLS ${ }^{M}$ modules and other established quality of life instruments

\begin{tabular}{|c|c|c|c|c|}
\hline & $Q_{L S}^{M}-A$ & $Q^{M} S^{M}-G$ & $Q_{L S}^{M}-M D$ & $Q^{M} S^{M}-D B S$ \\
\hline \multicolumn{5}{|l|}{ SF-36 MOS health scale } \\
\hline Physical component summary & 0.43 & 0.67 & 0.59 & 0.32 \\
\hline Mental component summary & 0.47 & 0.64 & 0.63 & 0.36 \\
\hline \multicolumn{5}{|l|}{ EQ-5D } \\
\hline EQ-5D index value & 0.49 & 0.67 & 0.68 & 0.46 \\
\hline EQ VAS score & 0.32 & 0.56 & 0.49 & 0.34 \\
\hline \multicolumn{5}{|l|}{ Questions on life satisfaction ${ }^{\text {modules }}$} \\
\hline General life satisfaction (QLSM $-A)$ & NA & NA & 0.53 & 0.50 \\
\hline Satisfaction with health (QLSM-G) & NA & NA & 0.75 & 0.62 \\
\hline Satisfaction with movement disorder (QLS $\left.{ }^{M}-M D\right)$ & NA & NA & NA & 0.65 \\
\hline Satisfaction with neurostimulation (QLS ${ }^{M}-D B S$ ) & NA & NA & 0.65 & NA \\
\hline
\end{tabular}

EQ-5D, EuroQol questionnaire ${ }^{17}$; NA, not applicable; SF-36, Medical outcome study 36 item short form health survey.

"deep brain stimulation." Table 3 shows the item characteristics including missing data, score distribution (means and standard deviations), summary scores, and floor and ceiling effects. Internal consistency is expressed as part-whole correlation of a single item with the scale value; reliability of the total scale is estimated by Cronbach's $\alpha$ (bold).

Considering that we were dealing with an impaired population, especially in the DBS treated group, the frequency of missing data was low. The mean item scores for weighted satisfaction were close to the mean of the possible range encompassing values from -12 to +20 . Floor and ceiling effects were negligible. The part-whole correlations between single item and the total scale mostly amounted between 0.4 and 0.6 , which was in the desired range.

\section{Validity}

Content validity was assumed to exist because of the assumptions on which the modules are based and the way the modules were developed, namely using patient generated issues from the initial exploratory interviews. Convergent validity was examined by means of correlating the new modules with established quality of life measures. Therefore we looked for agreement between the newly developed movement disorder and neurostimulation modules and the medical outcome study 36 item short form health survey (SF-36), ${ }^{16}{ }^{20}$ the EuroQol (EQ-5D), ${ }^{17}$ and the existing QLS ${ }^{\mathrm{M}}$ modules "general life satisfaction" and "satisfaction with health." ${ }^{17}$ Values of correlation coefficients are presented in table 4 .

Correlation coefficients for the convergent validity of the movement disorder module $\left(\mathrm{QLS}^{\mathrm{M}}-\mathrm{MD}\right)$ and SF36 reached values between 0.6 and 0.7 for the physical and mental health part of the instrument. Similar values were attained for the EQ-5D subdivided in the "EQ-5D index value" and a visual analogue scale. Somewhat lower values were found for the deep brain stimulation module (QLS ${ }^{\mathrm{M}}$-DBS). These lower measures were in line with our expectations, because the items of this module are quite specific to deep brain stimulation, and such issues are not included in instruments like the SF36 or the EQ-5D. Within the questions on life satisfactionmodules, correlation coefficients between the two established and the two new modules were between 0.5 and 0.7 .

\section{Translation}

The modules QLS-MD and QLS-DBS were transferred into US English with the help of forward translation and backward translation by two independent bilingual translators who are native speakers in the target language, as is standard in the transference of psychometric instruments to other countries. ${ }^{21}$

\section{DISCUSSION}

In this paper we describe the development and psychometric testing of a novel quality of life questionnaire to examine patients with movement disorders who had been or were to be treated with deep brain stimulation. Cronbach's $\alpha$ scores of 0.87 for $\mathrm{QLS}^{\mathrm{M}}$-MD and 0.73 for $\mathrm{QLS}^{\mathrm{M}}$-DBS indicate good reliability (above 0.5 is satisfactory, although ideally scores should exceed values of 0.7). ${ }^{22}$ Content validity was obtained by the use of patient generated issues from initial interviews. Construct validity was examined by means of correlations with SF-36 and EQ-5D. The self rated questionnaire was well accepted by the respondents and can be completed in less than 10 minutes even by disabled patients.

Our new questionnaire is based on an established multidimensional questionnaire concept - the questions on life satisfaction $^{\text {modules }}\left(\right.$ QLS $\left.^{\mathrm{M}}\right)$ - which has been validated in a representative sample of 7796 individuals in Germany, and has been translated into UK English, US English, Dutch, Spanish, and Italian. ${ }^{14}$ With a total of 16 items, QLS ${ }^{M}$ covers aspects of general life and health in two modules. This can now be combined with our two novel disease and intervention specific questionnaire modules, one for movement disorders (12 items) and one for neurostimulation (five items). Our approach with QLS $^{\mathrm{M}}$ differs from other disease specific, self rating questionnaires in that each item is weighted according to its relative importance to the individual. ${ }^{15}$ This is achieved using a weighting scale for each item: respondents are first asked to rate the subjective importance of a given area of life; then they are asked about the degree of satisfaction in that area.

The modules can be combined like a construction kit, depending on the study objective. For example, a DBS candidate receives modules I to III (general, health related, and movement disorder section) before operation, and after electrode implantation the same patient is asked to fill out all forms (I-III) including the DBS module (IV). In this way comparisons between preoperative and postoperative states and longitudinal studies become possible within the same QoL instrument and without loosing DBS specificity. The modules do not necessarily have to be used in a package. However, we do recommend this, as the combination of all modules covers comprehensive aspects of quality of life as proposed in the World Health Oranisation (WHO) position paper on general, health related, and disease specific QoL. ${ }^{23}$ The inclusion of all aspects of quality of life using different modules has an important advantage: depending upon study design, it allows for comparisons within a certain level of quality of life between healthy and affected individuals, between different diseases, and between different therapeutic interventions. Recently, a questionnaire for growth hormone replacement therapy was developed in a similar way and was 
successfully used in a modular approach together with the QLS $^{\mathrm{M}}$ modules for "general life" and "health". ${ }^{15}$ To our knowledge no study has dealt with the impact on quality of life of wearing, handling, and adverse events of neurostimulators, though this will be important when evaluating sophisticated and costly treatments which must not only be safe and effective according clinical rating scales, but should also lead to subjectively enhanced quality of life, at least for a substantial period of time and ideally for the rest of the patient's life.

We therefore looked for relevant items which represent subjectively assessed aspects of quality of life when neurostimulation is being given as a treatment for movement disorders. The following factors were crucial for quality of life in movement disorders: controllability and fluidity of movement, absence of dizziness, steadiness when standing or walking, hand dexterity throughout the day, articulation and fluency of speech, ability to swallow, absence of false bodily sensations, bladder and intestinal function, sexual excitability, undisturbed sleep, memory and clear thinking, independence from help, and inconspicuousness of illness. For satisfaction with deep brain stimulation, important features were the reliability, inconspicuousness, and controllability of the neurostimulator, the quality and availability of medical care, and the absence of bodily symptoms as side effects of the treatment. With the focus on neurostimulation, $\mathrm{QLS}^{\mathrm{M}}$-MD and $\mathrm{QLS}^{\mathrm{M}}$-DBS allow evaluation of perceived health status in nearly all patients in whom DBS is applied. To include Parkinson's disease, essential tremor, and idiopathic torsion dystonia in one QoL instrument has practical advantages, as in many former studies there was, at the least, a need to make comparisons between patients with Parkinson's disease and those with essential tremor. In the study by Schuurman et al, ${ }^{6}$ for instance, patients with tremor of different aetiology had to be studied with various different instruments, making comparisons with other reports and within the particular patient cohort difficult. In the future clinical studies on DBS in idiopathic torsion dystonia will become more frequent, and evaluation of quality of life in this field will gain more importance. ${ }^{9}$ Knowing that we need to cover a broad clinical field, we included data on Parkinson's disease, essential tremor, and dystonia during the development of the instruments. $\mathrm{QLS}^{\mathrm{M}}-\mathrm{MD}$ and $\mathrm{QLS}^{\mathrm{M}}-\mathrm{DBS}$ are validated for all three disease entities.

Mood and other positive or negative affective states have a major influence on the perception of symptoms and physical wellbeing. Thus QLS modules cover characteristics such as the ability to relax, inner peace, energy level, enjoyment of life, anxiety, sleep, sexuality, memory, and thinking. Nevertheless, the instrument is not validated to detect "depression" as a psychiatric disease. In studies where mood disorders are of substantial interest as a diagnostic entity, specific depression scales-for example, the Beck depression inventory ${ }^{24}$ or the MADRS $^{25}$ - should be added.

QLS $^{\mathrm{M}}$-MD and QLS ${ }^{\mathrm{M}}$-DBS are certainly a complement to and not a substitute for disease specific or even generic QoL scales. Future quality of life research in DBS will determine the role of subjective quality of life in relation to other instruments which do not include perceived health.

\section{CONCLUSIONS}

We present a syndrome specific measure of quality of life for patients treated with deep brain stimulation for movement disorders which has been comprehensively tested by psychometric methods. Within the established concept of questions on life satisfaction ${ }^{\text {modules }}\left(\right.$ QLS $\left.^{\mathrm{M}}\right)$ two new modules for movement disorders (QLS ${ }^{\mathrm{M}}$-MD) and deep brain stimulation $\left(\right.$ QLS $^{M}$-DBS) supplement the two existing basic modules for satisfaction with general life and health. The instrument is brief, well accepted by patients, and practical to administer in a clinic or as a postal questionnaire. We have shown that individual weighting of items is an effective and well accepted way of incorporating perceived health into a QoL instrument.

\section{ACKNOWLEDGEMENT}

The project was supported by the Deutsche Parkinson Vereinigung (DPV).

\section{Authors' affiliations}

A Kuehler, U Schroeder, B Conrad, A Ceballos-Baumann,

Department of Neurology, Technische Universität München, Munich, Germany

G Henrich, P Herschbach, Department of Psychosomatic Medicine, Technische Universität München

Competing interests: none declared

\section{REFERENCES}

1 Bain PG. Tremor assessment and quality of life measurements. Neurology 2000;54(suppl 4):S26-9.

2 Gudex CM, Hawthorne MR, Butler AG, et al. Effect of dystonia and botulinum toxin treatment on health-related quality of life. Mov Disord 1998;13:941-6.

3 Papathanasiou I, MacDonald L, Whurr R, et al. Perceived stigma in spasmodic torticollis. Mov Disord 2001;16:280-5.

4 Schrag A, Jahanshahi M, Quinn N. What contributes to quality of life in patients with Parkinson's disease? J Neurol Neurosurg Psychiatry 2000;69:308-12.

5 Benabid AL, Krack PP, Benazzouz A, et al. Deep brain stimulation of the subthalamic nucleus for Parkinson's disease: methodologic aspects and clinical criteria. Neurology 2000;55(suppl 6):S40-4.

6 Schuurman PR, Bosch DA, Bossuyt PM, et al. A comparison of continuous thalamic stimulation and thalamotomy for suppression of severe tremor. N Engl J Med 2000;342:461-8.

7 DBS study group. Deep-brain stimulation of the subthalamic nucleus or the pars interna of the globus pallidus in Parkinson's disease. N Engl J Med 2001;345:956-63.

8 Koller WC, Pahwa PR, Lyons KE, et al. Deep brain stimulation of the Vim nucleus of the thalamus for the treatment of tremor. Neurology 2000;55(suppl 6):S29-33.

9 Vercueil L, Pollak P, Fraix V, et al. Deep brain stimulation in the treatment of severe dystonia. J Neurol 2001;248:695-700.

10 Bullinger $M$. [Health related quality of life and subjective health. Overview of the status of research for new evaluation criteria in medicine]. Psychother Psychosom Med Psychol 1997;47:76-91.

11 Dodel RC, Berger K, Oertel WH. Health-related quality of life and healthcare utilisation in patients with Parkinson's disease: impact of motor fluctuations and dyskinesias. Pharmacoeconomics 2001;19:1013-38.

12 Straits-Troster K, Fields JA, Wilkinson SB, et al. Health-related quality of life in Parkinson's disease after pallidotomy and deep brain stimulation. Brain Cogn 2000;42:399-416.

13 Troster Al, Fields JA, Pahwa R, et al. Neuropsychological and quality of life outcome after thalamic stimulation for essential tremor. Neurology 1999:53:1774-80.

14 Henrich G, Herschbach P. Questions on life satisfaction (FLZM) - a short questionnaire for assessing subjective quality of life. Eur J Psychol Assess 2000; 16:150-9.

15 Herschbach P, Henrich G, Strasburger CJ, et al. Development and psychometric properties of a disease-specific quality of life questionnaire for adult patients with growth hormone deficiency. Eur J Endocrinol 2001;145:255-65.

16 McHorney CA, Ware JE, Raczek AE. The MOS 36-item short-form health survey (SF-36): II. Psychometric and clinical tests of validity in measuring physical and mental health constructs. Med Care 1993;31:247-63.

17 Brooks R. EuroQol: the current state of play. Health Policy 1996;37:53-72.

18 Larsen DK, Taylor S, Asmundson GJ. Exploratory factor analysis of the Pain Anxiety Symptoms Scale in patients with chronic pain complaints. Pain 1997;69:27-34.

19 SPSS. Copyright () 2002, SPSS Inc Headquarters, 233 S Wacker Drive, $11^{\text {th }}$ floor Chicago, Illinois 60606, 2002.

20 Ware JE, Sherbourne CD. The MOS 36-item short-form health survey (SF-36). I. Conceptual framework and item selection. Med Care 1992;30:473-83

21 Mathias SD, Fifer SK, Patrick DL. Rapid translation of quality of life measures for international clinical trials: avoiding errors in the minimalist approach. Qual Life Res 1994:3:403-12.

22 Peto V, Jenkinson C, Fitzpatrick R. PDQ-39: a review of the development, validation and application of a Parkinson's disease quality of life questionnaire and its associated measures. J Neurol 1998;245(suppl 1):S10-14.

23 WHO. The World Health Organisation quality of life assessment (WHOQOL): position paper from the World Health Organisation. Soc Sci Med 1995;41:1403-9.

24 Beck A, Ward C, Mendelson M, et al. An inventory for measuring depression. Arch Gen Psych 1961;4:53-63.

25 Montgomery SA, Asberg M. A new depression scale designed to be sensitive to change. Br J Psychiatry 1979;134:382-9. 